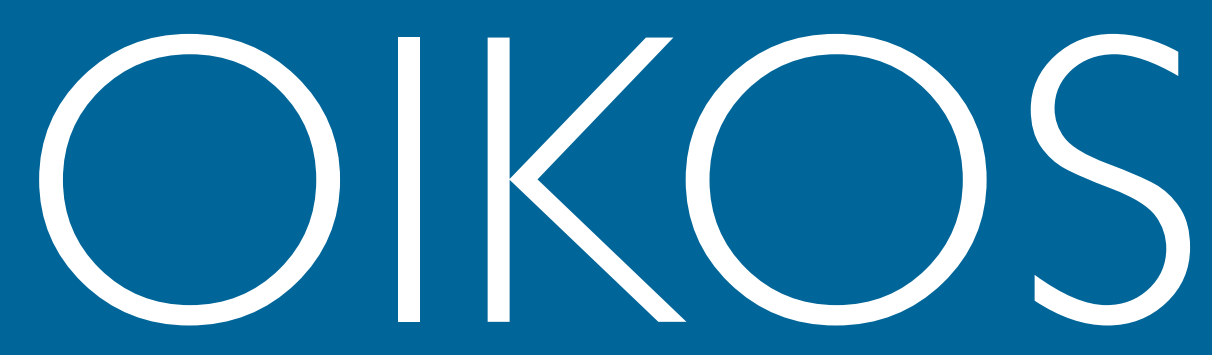

\title{
Research
}

\section{Fine-tuning the nested structure of pollination networks by adaptive interaction switching, biogeography and sampling effect in the Galápagos Islands}

\author{
Chinenye A. Nnakenyi, Anna Traveset, Ruben Heleno, Henintsoa O. Minoarivelo and Cang Hui
}

C. A. Nnakenyi (https:/lorcid.org/0000-0003-1009-5181) $\square$ (assumpta@sun.ac.za, chui@sun.ac.za), H. O. Minoarivelo and C. Hui, Centre for Invasion Biology, Dept of Mathematical Sciences, Stellenbosch Univ., Matieland 7602, South Africa. CH also at: Mathematical Biosciences Group, African Inst. for Mathematical Sciences, Cape Town, South Africa. - A. Traveset, Mediterranean Inst. of Advanced Studies (CSIC-UIB), Global Change Research Group, Esporles, Mallorca, Balearic Islands, Spain. - R. Heleno, Centre for Functional Ecology, Dept of Life Sciences, Univ. of Coimbra, Coimbra, Portugal.

\section{Oikos}

128: $1413-1423,2019$

doi: 10.1111/oik.06053

Subject Editor: Paulo Guimaraes Jr Editor-in-Chief: Dries Bonte Accepted 17 May 2019
The structure of pollination networks, particularly its nestedness, contain important information on network assemblages. However, there is still limited understanding of the mechanisms underlying nested pollination network structures. Here, we investigate the role of adaptive interaction switching (AIS), island area, isolation, age and sampling effort in explaining the nestedness of pollination networks across ten Galápagos Islands. The AIS algorithm is inspired by Wallace's elimination of the unfit, where a species constantly replaces its least profitable mutualistic partner with a new partner selected at random. To explain network structures, we first use a dynamic model that includes functional response of pollination and AIS, with only species richness and binary connectance as input (hereafter the AIS model). Thereafter, other explanatory variables (isolation, area, age and sampling effort) were added to the model. In four out of ten islands, the pollination network was significantly nested, and predictions from the AIS model correlated with observed structures, explaining 69\% variation in nestedness. Overall, in terms of independent contribution from hierarchical partitioning of variation in observed nestedness, the AIS model predictions contributed the most (37\%), followed by sampling effort (28\%) and island area (22\%), with only trivial contributions from island isolation and age. Therefore, adaptive switching of biotic interactions seems to be key to ensure network function, with island biogeographic factors being only secondary. Although large islands could harbour more diverse assemblages and thus foster more nested structures, sufficient sampling proves to be essential for detecting non-random network structures.

Keywords: adaptive rewiring, island biogeography, mutualism, nestedness, pollination network, sampling effort

\section{Introduction}

Islands often harbour highly endemic community assemblages that are largely constrained by biogeographical factors such as island area, age and degree of isolation (MacArthur and Wilson 1967). Besides these physical constraints, climate and human

(C) 2019 The Authors. Oikos (C) 2019 Nordic Society Oikos

www.oikosjournal.org 
disturbance can also largely affect insular ecological networks, particularly through biological invasions (Menke et al. 2012, Sebastián-González et al. 2015, Roura-Pascual et al. 2016, Tylianakis and Morris 2017). In particular, many plants and pollinators exploit the mutual benefits of cross-fertilisation and nectar harvest, improving the fitness of both partners (Bronstein 2015). As such, mutualistic interactions are key for enhancing and maintaining insular biodiversity and community functioning (Heleno et al. 2012, Traveset et al. 2016).

Mutualistic interactions, such as pollination or seed dispersal, often display patterns of nestedness (Lewinsohn et al. 2006, Bascompte and Jordano 2007, Bezerra et al. 2009, Fortuna et al. 2010, Sebastián-González et al. 2015). The degree of nestedness reflects the extent to which interactions are hierarchically arranged, so that species with less mutualistic partners (i.e. specialists) interact only with a proper subset of the partners of the more generalist species (Bascompte et al. 2003). As other network structures, the degree of nestedness of mutualistic networks is often scale dependent (Bezerra et al. 2009) and varies with sampling intensity (Blüthgen et al. 2007). For instance, subsets of a nested pollination network are often structured in a more nested way than the overall network (Bezerra et al. 2009). As such, nested structures become possible within the network motifs, and the level of nestedness is often rising at smaller scales (Lewinsohn et al. 2006, Flores et al. 2013). This scale dependence is often described using multilayer networks (Genrich et al. 2017, Pilosof et al. 2017).

Although a consensus is yet to be reached, nested networks seem to have important implications to the stability and persistence of mutualistic networks (Okuyama and Holland 2008, Thébault and Fontaine 2010, Staniczenko et al. 2010, Allesina and Tang 2012, James et al. 2012). In particular, it has been argued that highly nested mutualistic networks not only can support more species (Bastolla et al. 2009) but might also be more robust against habitat loss (Fortuna and Bascompte 2006) or species extinctions (Burgos et al. 2007). In contrast, it has also been argued that nestedness is only a by-product of species relative abundances rather than an actual driver of species coexistence (James et al. 2012) and network robustness (Minoarivelo and Hui 2016a). Although other network descriptors such as modularity have been found to decrease the stability of mutualistic communities (Thébault and Fontaine 2010), we confine our work solely to explaining observed levels of nestedness in mutualistic networks, as there exists an overall negative relationship between the levels of nestedness and modularity (Fortuna et al. 2010).

To explain structural emergence in ecological networks, earlier works have resorted to neutral processes such as random interactions between encountered individuals (Stang et al. 2006, Vázquez et al. 2007), and/or processes acknowledging the differences in species abundance or dispersal capacity (Lewinsohn et al. 2006). However, species abundances and encounter rates, as well as the strength of mutualistic interactions, are clearly not static, hinting at the need for a more dynamic model of adaptive network emergence. To this end, co-evolutionary processes have been proposed to affect not only the establishment of species interactions (Ehrlich and Raven 1964) but also the dynamics of ecological networks (Rezende et al. 2007a, Guimarães et al. 2011). Many studies have put forward different models and processes of network emergence based on evolutionary processes (Rezende et al. 2007b, Guimarães et al. 2011, Minoarivelo and Hui 2016b, 2018, Hui et al. 2017, Raimundo et al. 2018). However, although phylogenetic constraints on trait complementarity can influence interactions (Rezende et al. 2007b, Raimundo et al. 2018), they only account for a small amount of variation in network structure (Rezende et al. 2007b, Minoarivelo et al. 2014). To this end, evidence abounds that species can switch their interaction partners while searching for resources in both antagonistic and mutualistic networks (van Baalen et al. 2001, Kimbrell and Holt 2005, Basilio et al. 2006, Petanidou et al. 2008). For instance, generalist pollinators are constantly fine-tuning and rewiring their interaction partners to augment the accessible resources and, by doing so, shape the assembly of pollination networks (Ponisio et al. 2017). Such adaptive rewiring by animals can enhance the tolerance of mutualistic networks to species loss (RamosJiliberto et al. 2012). On a different temporal scale, species also inevitably need to rearrange their interactions when expanding on areas outside their native ranges - establishing novel interactions with resident species in invaded ecosystems (Traveset et al. 2013, Saul and Jeschke 2015, Le Roux et al. 2017, Hui and Richardson 2017, 2019), or after local extinction of co-occurring species (Costa et al. 2018). Such interaction rewiring can be very rapid, and is definitely much faster than evolutionary processes. As such, a model based on shortterm dynamical processes of interaction rewiring could drive network structure.

Ecological fitting, the formation of biotic interactions through compatibility of traits after rapid try-error matching, has been proposed as an alternative mechanism to coevolution for establishing novel biotic interactions (Janzen 1985, Raimundo et al. 2018). Ecological fitting is ultimately a process of rapid species rewiring - species adaptively switching and readjusting their interaction partners for short-term fitness gain regardless of whether they share any joint evolutionary history (Agosta and Klemens 2008). Because ecological fitting is also a sorting process, whereby only 'fits' can persist, its products resemble those that can be expected by coevolution (Agosta 2006). Previous studies have shown the importance of species rewiring in fostering species coexistence and persistence in species-rich or nutrientpoor communities (Murdoch 1969, Staniczenko et al. 2010, Valdovinos et al. 2010, Kaiser-Bunbury et al. 2010, RamosJiliberto et al. 2012, Suweis et al. 2013, but see Gilljam et al. 2015). Indeed, a species can switch partners based on the quality and quantity of available resources, as well as on the cost of acquiring such resources (Whittall and Hodges 2007, Valdovinos et al. 2010). Since mutualism is essentially a case of reciprocal exploitation, both species rewiring and ecological fitting can be framed under the theory of optimal and adaptive foraging, where species can adapt their diet based on current profitability, encounter rate and past experience 
(Stephens and Krebs 1986, Fossette et al. 2012, Zhang and Hui 2014). This provides a behavioural strategy for adaptive interaction switching (AIS), which has been implemented in a number of network-emergence models for explaining network structures in mutualistic communities (Kondoh 2003, Staniczenko et al. 2010, Zhang et al. 2011, Suweis et al. 2013, Mougi and Kondoh 2016).

Here, we first intend to predict pollination network structures in ten islands of the Galápagos Archipelago, using a model that implements adaptive interaction switching (AIS) based on Alfred Wallace's theory of natural selection via the elimination of the unfit, i.e. avoiding interactions with the less beneficial partners (Wallace 1864). In particular, the model uses observed species richness and network connectance as input, and adaptively reshuffles interactions between co-occurring species (Kondoh 2003, Zhang et al. 2011). We compare the results with a null model that maintains the marginal totals and connectance. Second, we intend to partition the amount of observed variation in network nestedness that is explained by the AIS model, by island biogeography theory (island area, isolation and age), and by sampling effort.

\section{Material and methods}

\section{Study system and observed nestedness}

The Galápagos Islands are located on the equator in the eastern Pacific Ocean, $960 \mathrm{~km}$ to the west of Ecuador in South America (Fig. 1). There are 18 main islands, with
San Cristóbal Island being the southernmost and the oldest (about $4 \mathrm{Ma}$ ), having an average island size of $141 \mathrm{~km}^{2}$. The youngest island, Fernandina, erupted between about 0.035 and $0.07 \mathrm{Ma}$ (Poulakakis et al. 2012), and Isabela, the largest and highest (1710 $\mathrm{m}$ a.s.l.) dates to approximately $0.75 \mathrm{Ma}$ (Ali and Aitchison 2014). About 30000 people live in the archipelago, concentrated mostly in Santa Cruz and San Cristóbal, although ca 2000 inhabit Isabela and ca 100 live in Floreana (Galápagos Conservancy 2016). Using Google Earth, we measured the shortest distances between the shores of each island and used the mean value in each group of measurement as the degree of isolation for each island.

The Galápagos flora consists of more than 1400 vascular plants, of which 59\% are aliens, $14 \%$ are endemic and $27 \%$ native (Jaramillo and Guézou 2013). Pollination data were collected from a survey of pollination events on 10 islands, including Fernandina, Pinta, San Cristóbal, Santa Cruz and Santiago (reported in Traveset et al. 2013; surveyed in February 2010 and 2011); and Española, Floreana, Genovesa, Isabela and Marchena (reported here; surveyed from 2012 to 2015). Visits of animals to open flowers were quantified by direct observation to randomly selected plants on all islands with variable sampling effort (min. 6, max. 126 hours per island) (Traveset et al. 2013; Table 1). All animals that contacted the reproductive organs of the flowers are hereafter referred as pollinators. The rationale and justification for the sampling design was presented elsewhere (Traveset et al. 2013).

Data from each island were compiled into a specific pollination network, with weighted interactions recorded

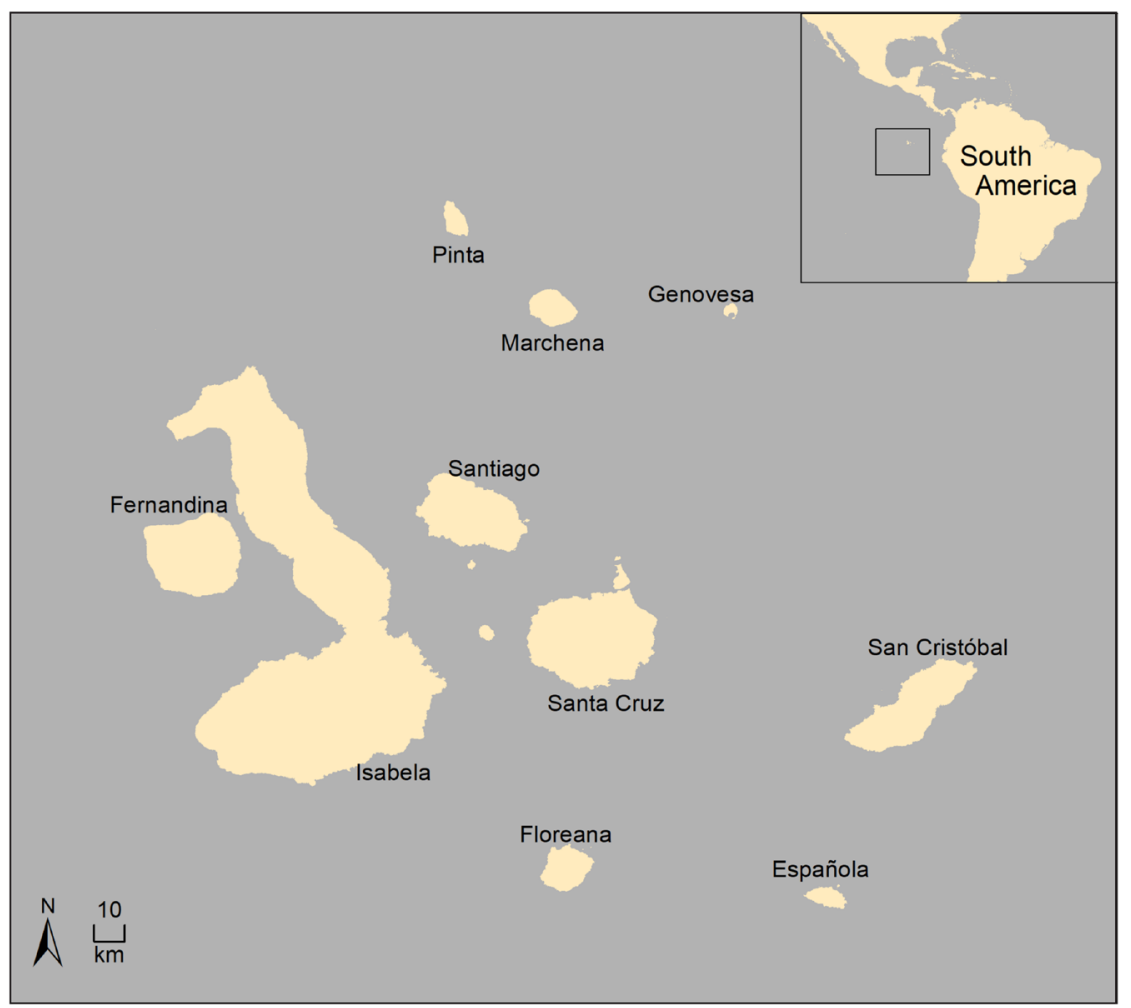

Figure 1. Map of the Galápagos Islands. Labels indicate the islands included in this study. 
Table 1. Characterization of the flower visitation networks and biogeographic variables of the 10 Galápagos Islands included in this study. $\mathrm{S}_{\mathrm{p}}, \mathrm{S}_{\mathrm{A}}$ and I are the number of plants, animals and interactions respectively; WC: weighted connectance; sampling effort is measured in hours; isolation, which is the average nearest distance of one island to the other is measured in $\mathrm{km}$; Island size is given by the area, measured in $\mathrm{km}^{2}$. Age is measured in millions of years (Ma) (Poulakakis et al. 2012, Galapagos Conservancy 2016).

\begin{tabular}{|c|c|c|c|c|c|c|c|c|}
\hline Islands & $S_{\mathrm{p}}$ & $\mathrm{S}_{\mathrm{A}}$ & 1 & WC & Sampling (h) & Isolation $(\mathrm{km})$ & Area $\left(\mathrm{km}^{2}\right)$ & Age (Ma) \\
\hline Española & 11 & 16 & 31 & 0.12 & 16.75 & 162.58 & 60 & 3.50 \\
\hline Fernandina & 18 & 60 & 125 & 0.06 & 70.25 & 128.00 & 642 & 0.07 \\
\hline Floreana & 11 & 4 & 13 & 0.25 & 6.30 & 125.85 & 173 & 2.30 \\
\hline Isabela & 12 & 16 & 28 & 0.13 & 23.42 & 75.65 & 4670 & 0.80 \\
\hline Marchena & 8 & 14 & 20 & 0.13 & 12.25 & 108.00 & 130 & 0.70 \\
\hline Pinta & 21 & 76 & 134 & 0.07 & 105.08 & 128.32 & 60 & 0.80 \\
\hline Santiago & 24 & 69 & 167 & 0.03 & 107.83 & 72.25 & 572 & 1.40 \\
\hline
\end{tabular}

as frequencies of flower visitations to focal flowers. The surveyed data allowed us to calculate the interaction matrix of visitation frequency $\left(V_{\mathrm{obs}}\right)$, weighted connectance (WC) and binary connectance (the proportion of non-zero elements of $V_{\mathrm{obs}}$ ) (Table 1). To measure to what degree specialist species tend to interact with particular subsets of the partners of more generalist species, we used the weighted nestedness metric WNODF (weighted nestedness based on overlap and decreasing fill; Almeida-Neto and Ulrich 2011), implemented using the $\mathrm{R}$ package bipartite 2.08 (Dormann et al. 2009) for R ver. 3.4.3 (<www.r-project.org>). Thereafter, we calculated the level of nestedness for the observed interaction matrix of visiting frequency $V_{\text {obs }}$.

To test the significance of the observed nestedness from the pollination networks, we compared the observed network structure with those generated at random from a null model. Since nestedness considers how specialist species interact with generalists, maintaining the number of interactions per species is important, and the null model for detecting nestedness should reflect this feature. We thus choose the null model of swap.web in the bipartite package. The null model reshuffles a given weighted matrix of interactions in such a way that maintains the matrix dimension, the number of links and the marginal totals, i.e. the number of links per species (Dormann et al. 2009). An observed network was said to be significantly nested when the observed value of nestedness was located within the $5 \%$ upper tail of 1000 null-model predictions.

\section{Adaptive interaction switching (AIS) model}

The population dynamics of resident species in a pollination network was simulated using a modified Lotka-Volterra model (Zhang et al. 2011). The model implements the mutualistic relationship between plant and animal species using Holling's (1959) type II functional response for reciprocal resource acquisition, and AIS of species interaction (Kondoh 2003, Zhang et al. 2011). Specifically, we depict the pollination network as a bipartite network of plants $(P)$ and animals $(A)$ for each island, where species in one partition interacts mutually with species in the other partition.
Therefore, for $m$ number of plants and $n$ number of animals, the population dynamics can then be described by the following (Okuyama and Holland 2008, Bastolla et al. 2009, Zhang et al. 2011, Suweis et al. 2013):

$$
\begin{aligned}
& \frac{d P_{i}}{d t}=r_{i}^{(P)} P_{i}-\alpha_{i}^{(P)} P_{i}^{2}+\frac{\sum_{j=1}^{n} a_{i j} \beta_{i j}^{(P)} A_{j} P_{i}}{1+h \sum_{k=1}^{n} a_{i k} A_{k}} \\
& \frac{d A_{j}}{d t}=r_{j}^{(A)} A_{j}-\alpha_{j}^{(A)} A_{j}^{2}+\frac{\sum_{i=1}^{m} a_{i j} \beta_{i j}^{(A)} P_{i} A_{j}}{1+b \sum_{k=1}^{m} a_{j k} P_{k}}
\end{aligned}
$$

where $P_{i}$ and $A_{j}$ are population densities of plant species $i$ and animal species $j$. Superscript $(P)$ and $(A)$ represents plant and animal species, respectively.

From the right-hand side of both equations above, the first term depicts population growth, with $r_{i}^{(P)}$ and $r_{j}^{(A)}$ representing the intrinsic growth rates of plant species $i$ and animal species $j$. The second term incorporates the densitydependence, where $\alpha_{i}^{(P)}$ and $\alpha_{j}^{(A)}$ are the coefficients. The third term is the functional response that describes the fitness gain from the mutualistic interactions, where $a_{i j}$ is the element on row $i$ and column $j$ of the binary interaction matrix $M_{m \times n}$, and indicates whether plant $i$ interacts with animal $j$ $\left(a_{i j}=1\right)$ or not $\left(a_{i j}=0\right)$. Parameters $\beta_{i j}^{(P)}$ and $\beta_{i j}^{(A)}$ describe the per-capita benefits obtained per unit of time by plant $i$ from interacting with animal $j$ and by animal $j$ from pollinating plant $i$, respectively. The parameter $h$ stands for the handling time, representing the proportion of time a species spends on searching and handling resources (i.e. not all time was used for consuming resources).

To implement AIS, where a species can adaptively rewire its interacting partners based on the elimination of the unfit algorithm (Zhang et al. 2011), we followed a two-step procedure: eliminating the least contributing partner and rewiring to a randomly selected partner. In particular, at each time step when numerically solving the above equations, a species is selected at random. For example, say animal $j$ is selected, we then evaluate the relative benefit contribution received from interacting with a plant species by comparing 
$a_{i j} \beta_{i j}^{(A)} P_{i}$ for $i=1, \ldots, m$, and identify the plant species that interacts with animal $j$ (those $a_{i j}=1$ ) but contributes the least (say, plant $k$ ) to animal $j$ 's benefit/fitness gain (thus the one with the minimum nonzero relative contribution). Once identified, animal $j$ then stops its interaction with plant $k$, by setting $a_{k j}$ from 1 to 0 . We then choose a non-interacting plant species at random, say plant $l$ (those currently with zero relative contribution, $a_{l j}=0$ ), and switch the interaction between animal $j$ and plant $k$ to between animal $j$ and plant $l$ by setting $a_{k j}=0$ and $a_{i j}=1$.

\section{Numerical simulation and parameterization}

We conducted numerical simulations, solving the ordinary differential equations with the Runge-Kutta order 4 method at a step size of 0.01 , using deSolve package in $\mathrm{R}$ (Soetaert et al. 2010). At each time step, the AIS switching algorithm is implemented, and network structure computed (see Supplementary material Appendix 1 for R scripts). The initial interaction matrix $a_{i j}$ were randomly assigned as a binary $0 / 1$ matrix of dimension $m \times n$ with $c$ proportion of elements being 1 and the rest 0 , where $m$ represents the number of plant species, $n$ the number of animal species and $c$ the binary connectance observed. Other model parameters were randomly assigned from uniform and normal distributions. Specifically, we assigned initial population densities, growth rates and density dependent coefficients from the uniform distribution $U(0,1)$; per capita benefits $\beta_{i j}^{(P)}$ and $\beta_{i j}^{(A)}$ were assigned from the nonnegative normal distribution $|N(0,0.05)|$. For simplicity, the same handling time was assigned for all species, $b=0.1$. The selection of the specific bounds of parameter distributions was simply to ensure the persistence of all species. Other bounds that still ensure the persistence of all species do not alter the results qualitatively (and thus are not shown).

Therefore, for each island we used three observed numbers ( $m, n$ and $c$ ) as model input and generated the encountering matrices $V_{\mathrm{AIS}}=\left\langle a_{i j} P A_{j}\right\rangle_{m \times} n$ for each of the last 1000 time steps (from a total 5000 time steps of running the model). We calculated the nestedness of encountering matrices also using WNODF. Due to the stochasticity associated with assigning parameters from random distributions (especially for small networks) and the lack of empirical estimates of population growth, density dependence and mutualistic benefits, we ran 100 replicates of the model for each island. We then chose the set of parameters that generated the closest average nestedness of the last 1000 encountering matrices to the nestedness of observed visiting frequency matrix. From this semi-optimisation procedure of parameterisation, albeit still randomly drawn from the same specified probability distributions, the chosen parameter set should reflect, to some degree, the context- and island-dependent strengths of pairwise biotic interactions. That is, this procedure is a compromised solution to addressing the missing information on the population growth, density dependence and mutualistic benefits of the species on each island, which are required for running the model.
To assess how much variation in the observed nestedness can be explained by the AIS model, we first performed a linear regression of predicted versus observed values for each network, using reduced major axis regression from the $\mathrm{R}$ package lmodel2 1.7-2 (Legendre 2014). In addition to the AIS model predictions, other variables such as sampling effort, island area, isolation and age, were combined to predict the observed network structures. Consequently, we checked for collinearity among these explanatory variables using variance inflation factor (VIF) in the $\mathrm{R}$ package fmsb 0.6.1 (Nakazawa 2017), keeping only variables with VIF values lower than 10 . Thereafter, the combined variables were used to explain the observed nestedness using generalised linear model (GLM) in R.

Due to the low number of networks (i.e. 10 islands), and to avoid overfitting, we further ran cross-validation by fitting the GLM using data from nine islands and predicting the network structure of the tenth island. Because of the semioptimisation procedure of parameterisation and the potential of overfitting, the importance of AIS algorithm could have potentially been inflated (although not necessarily so). To address this problem, we partitioned the independent and conjoint contribution of these explanatory variables using the hierarchical variance partitioning method from the $\mathrm{R}$ package hier.part 1.0-4 (Walsh and Mac Nally 2013). The independent contribution, by assessing the reduction of GLM performance from removing a variable, should thus reflect the minimum or conservative role of the AIS algorithm in explaining observed levels of nestedness. In addition, the difference between the conjoint and independent contribution should reflect the inflation from the procedure of parameterisation, interactions and correlations between explanatory variables, and potential overfitting.

\section{Results}

\section{Nestedness from AIS model}

The pollination networks assembled on four out of the ten islands were significantly nested (Table 2). In contrast, none of the ten pollination networks were significantly modular (Supplementary material Appendix 2 Table A2.1 and Fig. A2.1). From the AIS model simulations, the final number of species in the predicted networks (Fig. 2a) was the same as in the observed networks (Fig. 2b), i.e. no species became extinct (Fig. 2c-d). For each island, a specific set of parameters were chosen through the semi-optimisation procedure of parameterisation. The nestedness predicted by the AIS models gradually converged to the value of the observed nestedness, irrespective of the network's initial structure (Fig. 3), and so the AIS model was not rejected for six pollination networks: Española, Isabela, Marchena, Pinta, Santa Cruz and Santiago (Fig. 4). According to the RMA regression, the AIS model predictions greatly correlated with observed nestedness $\left(\mathrm{R}^{2}=0.69, \mathrm{p}=0.003\right.$, RMA slope $=0.50)($ Fig. 4). 
Table 2. Nestedness of the pollination networks of 10 Galápagos Islands, predicted by adaptive interaction switching (AIS) and null model. Each value of the model corresponds to the 95\% confidence interval $\left(\bar{X} \pm Z_{\alpha / 2} \sigma_{\bar{X}}\right)$, where $\bar{X}$ and $\sigma_{\bar{X}}$ are the mean and standard deviation of the model predictions respectively; $Z_{\alpha / 2}=1.96$ is the critical value; number of samples is 1000 for both the AIS model and the null model. The observed values in bold are significantly nested, while the underlined model predictions are not significantly different from the observed values.

\begin{tabular}{lccc}
\hline & & \multicolumn{2}{c}{ Model predictions } \\
\cline { 3 - 4 } Islands & Observed & AIS & Null \\
\hline Española & $\mathbf{0 . 0 8 2}$ & $\underline{0.116 \pm 0.045}$ & $0.073 \pm 0.040$ \\
Fernandina & 0.092 & $0.109 \pm 0.015$ & $0.213 \pm 0.045$ \\
Floreana & 0.000 & $0.081 \pm 0.078$ & $0.029 \pm 0.069$ \\
Genovesa & 0.053 & $0.074 \pm 0.017$ & $0.057 \pm 0.023$ \\
Isabela & $\mathbf{0 . 1 6 3}$ & $\underline{0.109 \pm 0.063}$ & $0.143 \pm 0.058$ \\
Marchena & $\mathbf{0 . 0 5 0}$ & $\underline{0.074 \pm 0.047}$ & $0.050 \pm 0.037$ \\
Pinta & 0.104 & $\underline{0.091 \pm 0.017}$ & $0.147 \pm 0.026$ \\
San Cristóbal & $\mathbf{0 . 1 8 2}$ & $0.158 \pm 0.015$ & $0.174 \pm 0.018$ \\
Santa Cruz & 0.160 & $\underline{0.160 \pm 0.020}$ & $0.223 \pm 0.027$ \\
Santiago & 0.133 & $\underline{0.133 \pm 0.014}$ & $0.203 \pm 0.029$ \\
\hline
\end{tabular}

All the explanatory variables including the AIS model predictions, the geographical factors (island area, isolation, age) and sampling effort were found to be independent variables, significantly contributing for explaining nestedness (Supplementary material Appendix 3 Table A3.1). Hence, the GLM model combining all independent variables explained more than $90 \%$ of the variation in observed nestedness (i.e. $\mathrm{R}^{2}=0.92, \mathrm{p}<0.0001$ ). However, the correlation between observed and cross-validated nestedness was weak $(\mathrm{r}=-0.072, \mathrm{p}=0.844)$, indicating overfitting. As such, we relied on the independent contribution of each variable from the hierarchical partitioning as a conservative indicator of its role in explaining observed nestedness, whereas the difference between conjoint and independent contribution reflected the inflated contribution due to overfitting and other issues. Albeit the noteworthy difference between conjoint and independent contribution (Fig. 5, Supplementary material Appendix 3 Table A3.2), the AIS model as an explanatory variable remained the greatest independent contributor $(37.27 \%)$, followed by sampling effort $(27.76 \%)$ and island area $(22.11 \%)$, whereas island isolation and age explained each less than $10 \%$ of the variation in observed nestedness.

\section{Discussion}

\section{Adaptive rewiring}

By using the algorithm for adaptive interaction switching, we could to some extent predict particularly the structure of those nested insular pollination networks on the Galápagos Islands (Table 2). Overall, when evaluated alone, the AIS (a)

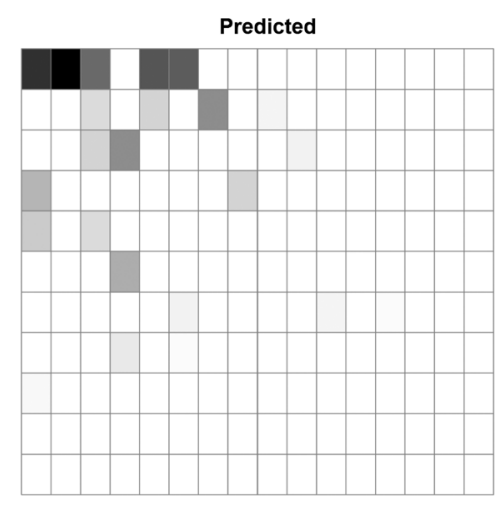

(c)

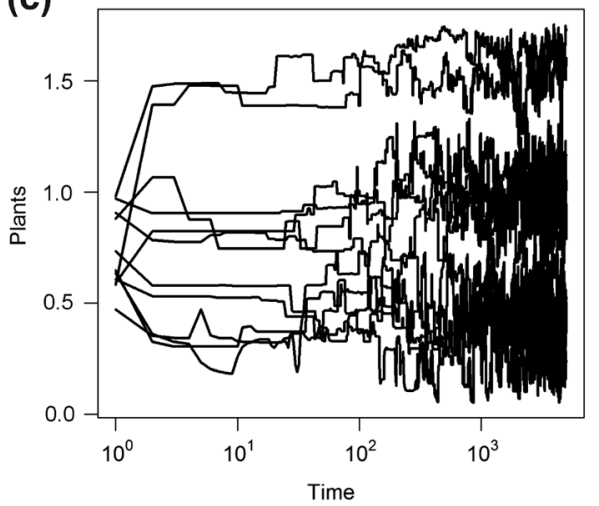

(b)

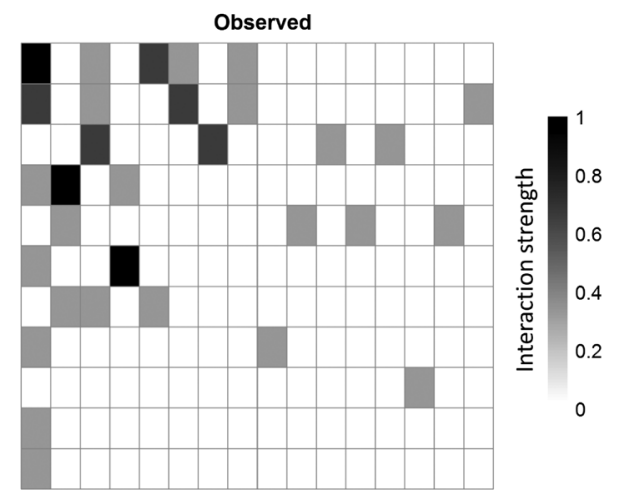

(d)

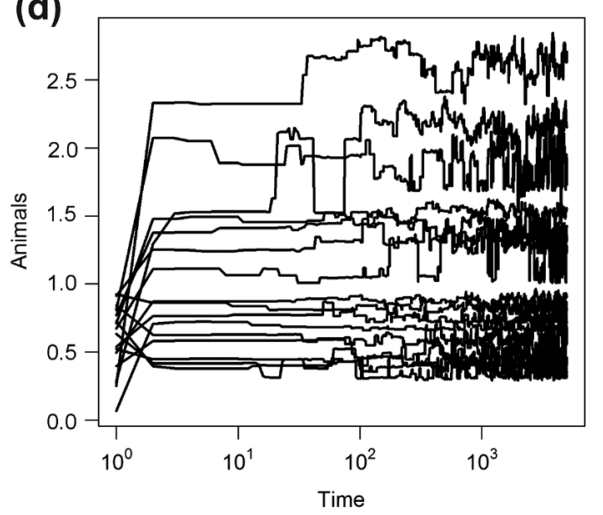

Figure 2. Data from the Española Island showing (a) predicted interaction matrix, (b) observed interaction matrix, (c) plant and (d) animal population abundances over the simulation time from the AIS model. 

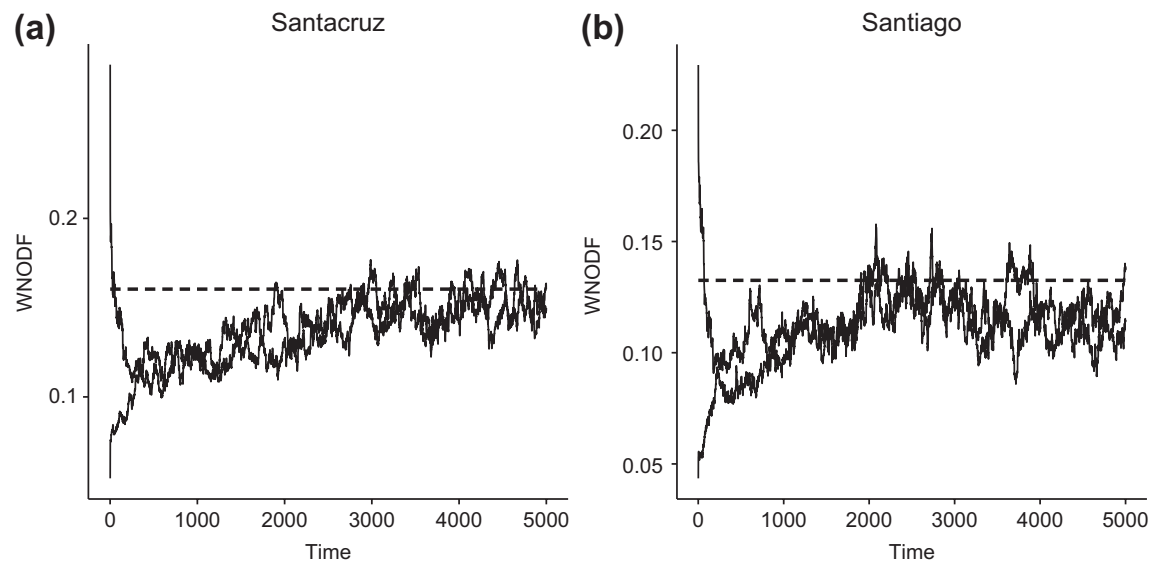

Figure 3. AIS model predictions of nestedness from a high and low initial network structure using (a) Santa Cruz and (b) Santiago Islands. The broken line is the observed value for each of the islands' data.

model captured almost $70 \%$ of the cross-island variation in nestedness, supporting the claim that adaptive interaction switching, not random rewiring (captured by the null model), is an important process of structural emergence in

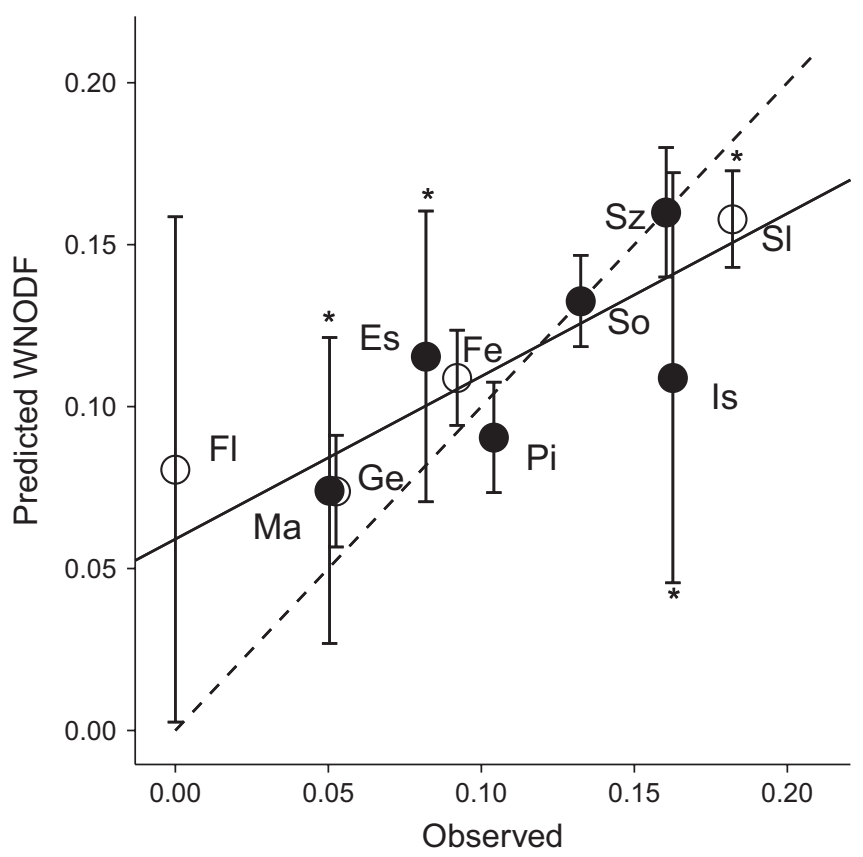

Figure 4. Reduced major axis regression (RMA) of the predicted versus observed nestedness (WNODF) of 10 pollination networks from the Galápagos Islands. The predictions are obtained from the AIS model. The black line is the RMA line with a slope of 0.50 $\left(\mathrm{R}^{2}=0.69, \mathrm{p}=0.003\right)$. Each circle represents an island network where the filled circles represents the networks that are not significantly different from their observed values, and the open ones are significantly different from their observed values. Asterisked bars are significantly nested. The broken line is for $\mathrm{y}=\mathrm{x}$. Island names are Es - Espańola, Fe - Fernandina, Fl - Floreana, Ge - Genovesa, Is Isabela, Ma - Marchena, Pi - Pinta, Sl - San Cristóbal Sz - Santa Cruz, So - Santiago. ecological networks (Kondoh 2003, Valdovinos et al. 2010, Zhang et al. 2011, Suweis et al. 2013, Nuwagaba et al. 2015). To further explore the role of adaptive rewiring to network stability, we estimated the asymptotic stability using the lead eigenvalue of the encountering matrix and found that adaptive rewiring could destabilise a system by pushing it towards marginal instability (Supplementary material Appendix 5 Fig. A5.1; Hui and Richardson 2019). In particular, we discovered a positive correlation between nestedness and the lead eigenvalue from adaptive switching, but a negative correlation when switching was not allowed (Supplementary material Appendix 5 Fig. A5.2). This is consistent with the notion of Suweis et al. (2013) that the cost to an optimised network from adaptive rewiring is its reduced stability. Therefore, adaptive rewiring can greatly affect network stability and function, while the pattern of nestedness could simply be a by-product of marginal instability (Hui and Richardson 2019).

Conserving and restoring pollination interactions can be crucial for the functioning of island ecosystems (KaiserBunbury et al. 2010) as keystone species often foster strong asymmetric interactions and thus produce mostly nested structures in such isolated ecosystems (Dupont et al. 2003). Indeed, in contrast to significant nestedness in most insular pollination networks at the local island scale, none was significantly modular (Supplementary material Appendix 2 Table A2.1). However, on a larger scale, the pattern of modularity is visible at the archipelago scale (Traveset et al. 2013, using a subset of the datasets presented here as a single meta-network). This is consistent with the observation that relatively small pollination networks could hardly exhibit significant levels of modularity (Olesen et al. 2007), as well as the multiscale and multilayer nature of mutualistic networks (Genrich et al. 2017, Pilosof et al. 2017). As such, we argue that the AIS process is crucial to understanding nonrandom network structure; however, when network structure is indistinguishable from random, the contribution of the AIS process to network emergence inevitably declines. 


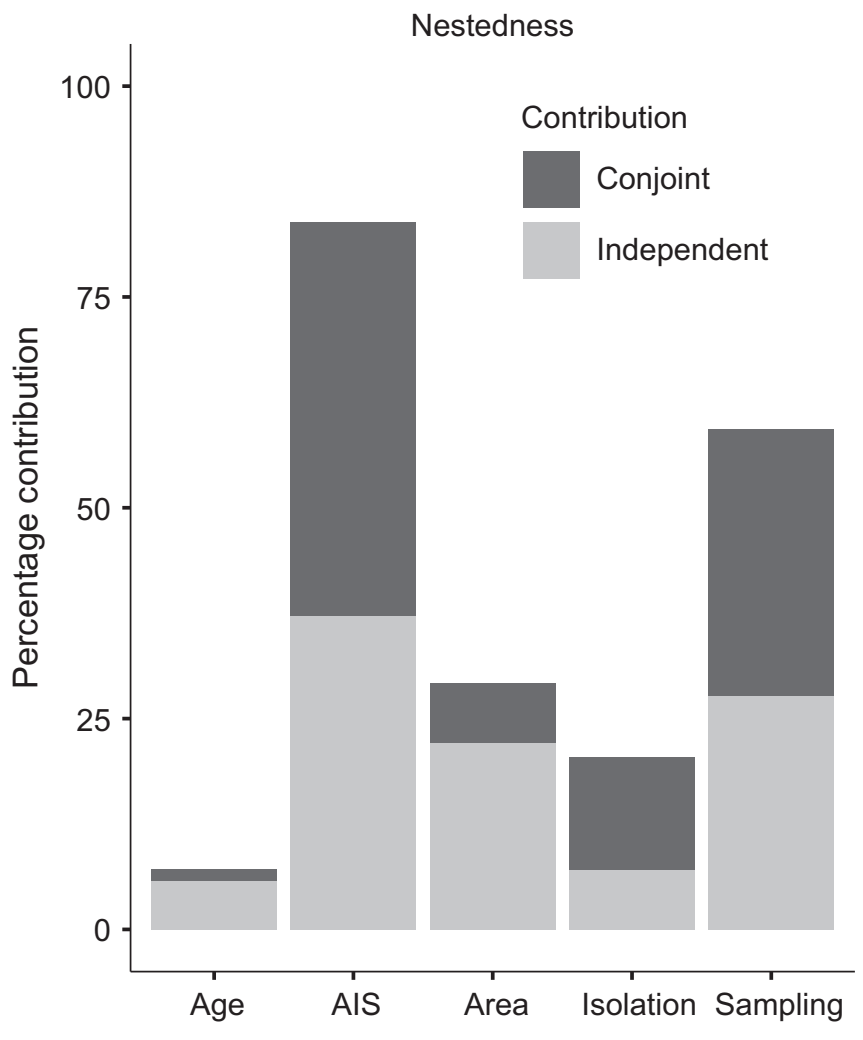

Figure 5. Nestedness and variance hierarchical partitioning showing the percentage of the independent and conjoint contribution of each of the explanatory variables: AIS model, age, area, isolation and sampling effort.

\section{Sampling effect}

The number of pollination events recorded on each island was found to correlate with the sampling effort $\left(R^{2}=0.91\right.$, $\mathrm{p}<0.001, y=1.72 \mathrm{x}+0.03$; Supplementary material Appendix 4 Fig. A4.1). When resampling an artificial large network (50 plants and 200 pollinator species, generated using the AIS model) under different levels of sampling effort, we discovered a threshold for detecting its non-random structures, after observing more than 60 pollination events (equivalent to 34.9 h) (Supplementary material Appendix 4 Fig. A4.2). With only 20-60 observed pollination events (11.6-34.9h), sampling incompleteness can strongly hinder our capacity to detect relevant patterns on network structure, likely underestimating nestedness (Supplementary material Appendix 4 Fig. A4.2, A4.3). As a result, sampling effort was positively correlated with nestedness for the insular pollination networks $(r=0.609$, $\mathrm{p}=0.06$ ). Accordingly, sampling effort was the second best predictor for explaining nestedness (Fig. 5). Unlike Trøjelsgaard and Olesen (2013), where sampling effort explained $<1 \%$ of the variation in nestedness in pollination networks, we found that sampling effort independently contributed $28 \%$ in explaining the cross-island nestedness pattern (Fig. 5). This is aligned with other studies that acknowledge the importance of sampling effect in detecting nestedness and other network structures (Blüthgen et al. 2007, Blüthgen 2010).
In contrast, however, even with sufficient sampling effort we did not discover any modular patterns at local island scales, suggesting that the pattern of compartmentalisation could be completely lacking within an island pollination network. This, however, could also mean that modularity is much more difficult to detect than nestedness on a local scale. This finding is in agreement with Vizentin-Bugoni et al. (2015) but contrasts with previous findings that nestedness is not highly sensitive to sampling completeness (Nielsen and Bascompte 2007). With the increase of sampling effort (Supplementary material Appendix 4 Fig. A4.2, A4.3), we could expect to discover more rare and aggregated species, as well as rare interactions which could alter interaction patterns and shift the distribution of interaction strength (Etienne and Alonso 2005). Consequently, sampling effort should continue to be regarded as a crucial factor to consider when comparing network structure (Rivera-Hutinel et al. 2012, Costa et al. 2016, Falcão et al. 2016).

\section{Island biogeography}

The emergence of structural patterns on the pollination networks on the Galápagos Islands is secondarily driven by biogeographical factors. Besides the AIS process and sampling effects, island area was also found to be an important driver of nestedness across islands, though the level of island isolation was the highest conjoint contributor of modularity across islands (Supplementary material Appendix 2 Fig. A2.2, Table A2.2). This is in line with RouraPascual et al. (2016) that island isolation is a plausible explanation for the spatial modularity of native ant assemblages. Similarly, large islands harbour more heterogeneous habitats and thus more diverse assemblages, allowing species to diversify their interacting partners and interaction promiscuity (Ricklefs and Lovette 1999, Montoya et al. 2015), fostering nested network structures. Taken together, biogeographical factors only partially explain the interaction patterns on local species assemblages, whereas the network structure emerges from each species surviving and negotiating in the games and tradeoffs with other members of the network (Nuwagaba et al. 2015, Pinheiro et al. 2016, Minoarivelo and Hui 2018). These games and tradeoffs, together with direct and indirect biotic interactions, shape the trait evolution in an ecological network (Guimarães et al. 2017, Hui and Richardson 2019), which in return also affects species interactions and abundances (Raimundo et al. 2018). It further suggests the need to consider trait matching and trait coevolution in future model of adaptive networks, aiming at capturing the structure and function of mutualistic networks (Traveset et al. 2013, Chamberlain et al. 2014, Minoarivelo and Hui 2016b). Biogeographical factors are important to network assembly, but only secondary to network structure and function which arise largely from the multiplayer games of resident species.

The reason that a previous study using datasets from five of the Galápagos Islands detected a significant modularity pattern (Traveset et al. 2013) could be because 
a binary metric was used and the data was combined to form a large scale meta-network at the archipelago level, instead of considering the islands as separate networks. In so doing, several of the detected modules at the archipelago level largely coincided with specific island assemblages. This confirms the scale dependency of network modularity that often emerges at larger geographic and/or taxonomic scales, in contrast to nestedness that becomes more notable at local scales (Lewinsohn et al. 2006, Flores et al. 2013). This suggests that, first, modularity patterns in real mutualistic networks could reflect multiple subnetworks that are divided by dispersal barriers, and are thus connected by limited dispersal - our result on island isolation being the second most important contributor to the cross-island variation in modularity further support this proposition (Supplementary material Appendix 2 Fig. A2.2; see also Timóteo et al. 2018). The implementation of spatially explicit multilayer modularity (Pilosof et al. 2017, Timóteo et al. 2018), recognising the interdependence of islands within an archipelago context, holds great promise to further understand the implications of biogeography, including island isolation, on archipelago-level meta-community structure. Nevertheless, such approach would ideally require an independent quantification of inter-island connectivity for each species, which remains a heady goal. We expect that at increasing spatial scales (from local to meta-network), a transition from a predominantly nested structure to a more modular pattern should be observed in mutualistic meta-networks.

Overall, we show that AIS seems a key driving mechanism behind community nestedness, with a secondary role of biogeographic factors. However, sampling effort needs to be explicitly considered when exploring the drivers of network structure as there is a minimum sampling threshold below which some patterns will likely go undetected.

Acknowledgements - We are grateful to Feng Zhang, Pietro Landi, David M. Richardson, Ulf Dieckmann and Åke Brännström for constructive discussions on this topic. Also to Jens M. Olesen, Pablo Vargas and Manuel Nogales, as well as all the members during the field trip, for data collection of the pollination networks.

Funding - CAN receives a PhD scholarship from the Southern African Systems Analysis Centre (SASAC) and Deutscher Akademischer Austausch Dienst (DAAD: German Academic Exchange Service). HOM acknowledges support from DST-NRF Centre of Excellence in Mathematical and Statistical Sciences (CoE-MaSS; BA2017/136). CH was supported by the National Research Foundation of South Africa (89967 and 109244) and the Australian Research Council (Discovery Project DP150103017). AT and RH were supported by the Spanish Government (CGL2017$88122-\mathrm{P})$ and by the Portuguese Foundation for Science and Technology (IF/00441/2013), respectively.

Author contributions - CAN and CH conceived the study, AT and $\mathrm{RH}$ collated the data, CAN, HOM and $\mathrm{CH}$ analysed the data, and all authors contributed to the writing and editing of the manuscript.

\section{References}

Agosta, S. J. 2006. On ecological fitting, plant-insect associations, herbivore host shifts and host plant selection. - Oikos 114: 556-565.

Agosta, S. J. and Klemens, J. A. 2008. Ecological fitting by phenotypically flexible genotypes: implications for species associations, community assembly and evolution. - Ecol. Lett. 11: 1123-1134.

Ali, J. R. and Aitchison, J. C. 2014. Exploring the combined role of eustasy and oceanic island thermal subsidence in shaping biodiversity on the Galápagos. - J. Biogeogr. 41: 1227-1241.

Allesina, S. and Tang, S. 2012. Stability criteria for complex ecosystems. - Nature 483: 205-208.

Almeida-Neto, M. and Ulrich, W. 2011. A straightforward computational approach for measuring nestedness using quantitative matrices. - Environ. Model. Softw. 26: 173-178.

Bascompte, J. and Jordano, P. 2007. Plant-animal mutualistic networks: the architecture of biodiversity. - Annu. Rev. Ecol. Evol. Syst. 38: 567-593.

Bascompte, J. et al. 2003. The nested assembly of plant-animal mutualistic networks. - Proc. Natl Acad. Sci. USA 100: 9383-9387.

Basilio, A. M. et al. 2006. A year-long plant-pollinator network. - Austral Ecol. 31: 975-983.

Bastolla, U. et al. 2009. The architecture of mutualistic networks minimizes competition and increases biodiversity. - Nature 458: 1018-1020.

Bezerra, E. L. S. et al. 2009. Pollination networks of oil-flowers: a tiny world within the smallest of all worlds. - J. Anim. Ecol. 78: 1096-1101.

Blüthgen, N. 2010. Why network analysis is often disconnected from community ecology: a critique and an ecologist's guide. - Basic Appl. Ecol. 11: 185-195.

Blüthgen, N. et al. 2007. Specialization, constraints and conflicting interests in mutualistic networks. - Curr. Biol. 17: 341-346.

Bronstein, J. L. 2015. Mutualism. - Oxford Univ. Press.

Burgos, E. et al. 2007. Why nestedness in mutualistic networks? - J. Theor. Biol. 249: 307-313.

Chamberlain, S. A. et al. 2014. Traits and phylogenetic history contribute to network structure across Canadian plantpollinator communities. - Oecologia 176: 545-556.

Costa, J. M. et al. 2016. Sampling completeness in seed dispersal networks: when enough is enough. - Basic Appl. Ecol. 17: $155-164$.

Costa, J. M. et al. 2018. Rewiring of experimentally disturbed seed dispersal networks might lead to unexpected network configurations. - Basic Appl. Ecol. 30: 11-22.

Dormann, C. F. et al. 2009. Indices, graphs and null models: analyzing bipartite ecological networks. - Open Ecol. J. 2: 7-24.

Dupont, Y. L. et al. 2003. Structure of a plant-flower-visitor network in the high-altitude sub-alpine desert of Tenerife, Canary Islands. - Ecography 26: 301-310.

Ehrlich, P. R. and Raven, P. H. 1964. Butterflies and plants: a study in coevolution. - Evolution 18: 586-608.

Etienne, R. S. and Alonso, D. 2005. A dispersal-limited sampling theory for species and alleles. - Ecol. Lett. 8: 1147-1156.

Falcão, J. C. et al. 2016. Sampling effort differences can lead to biased conclusions on the architecture of ant-plant interaction networks. - Ecol. Complexity 25: 44-52. 
Flores, C. O. et al. 2013. Multi-scale structure and geographic drivers of cross-infection within marine bacteria and phages. - ISME J. 7: 520-532.

Fortuna, M. A. and Bascompte, J. 2006. Habitat loss and the structure of plant-animal mutualistic networks. - Ecol. Lett. 9: 278-283.

Fortuna, M. A. et al. 2010. Nestedness versus modularity in ecological networks: two sides of the same coin? - J. Anim. Ecol. 79: 811-817.

Fossette, S. et al. 2012. Does prey size matter? Novel observations of feeding in the leatherback turtle (Dermochelys coriacea) allow a test of predator-prey size relationships. - Biol. Lett. 8: 351-354.

Galápagos Conservancy 2016. The islands. - <www.galapagos.org/ about_galapagos/about-galapagos/the-islands $>$.

Genrich, C. M. et al. 2017. Duality of interaction outcomes in a plant-frugivore multilayer network. - Oikos 126: 361-368.

Gilljam, D. et al. 2015. Adaptive rewiring aggravates the effects of species loss in ecosystems. - Nat. Commun. 6: 8412.

Guimarães, P. R. et al. 2011. Evolution and coevolution in mutualistic networks. - Ecol. Lett. 14: 877-885.

Guimarães Jr, P. R. et al. 2017. Indirect effects drive coevolution in mutualistic networks. - Nature 550: 511-514.

Heleno, R. H. et al. 2012. Seed dispersal networks in the Galápagos and the consequences of alien plant invasions. - Proc. R. Soc. B 280: 20122112.

Holling, C. S. 1959. Some characteristics of simple types of predation and parasitism. - Can. Entomol. 91: 385-398.

Hui, C. and Richardson, D. M. 2017. Invasion dynamics. - Oxford Univ. Press.

Hui, C. and Richardson, D. M. 2019. How to invade an ecological network. - Trends Ecol. Evol. 34: 121-131.

Hui, C. et al. 2017. Modelling coevolution in ecological networks with adaptive dynamics. - Math. Meth. Appl. Sci. 41: 8404-8422.

James, A. et al. 2012. Disentangling nestedness from models of ecological complexity. - Nature 487: 227-230.

Janzen, D. H. 1985. On ecological fitting. - Oikos 45: 308-310.

Jaramillo, D. P. and Guézou, A. 2013. CDF checklist of galápagos vascular plants. - In: Bungartz, F. et al. (eds), Charles Darwin Foundation galapagos species checklist. Charles Darwin Foundation, Puerto Ayora, Ecuador.

Kaiser-Bunbury, C. N. et al. 2010. The robustness of pollination networks to the loss of species and interactions: a quantitative approach incorporating pollinator behaviour. - Ecol. Lett. 13: 442-452.

Kimbrell, T. and Holt, R. D. 2005. Individual behaviour, space and predator evolution promote persistence in a two-patch system with predator switching. - Evol. Ecol. Res. 7: 53-71.

Kondoh, M. 2003. Foraging adaptation and the relationship between food-web complexity and stability. - Science 299: 1388-1391.

Le Roux, J. J. et al. 2017. Co-introduction vs ecological fitting as pathways to the establishment of effective mutualisms during biological invasions. - New Phytol. 215: 1354-1360.

Legendre, P. 2014. Imodel2: model II regression. - R package ver. 1.7-2. <https://CRAN.R-project.org/package=lmodel2>.

Lewinsohn, T. M. et al. 2006. Structure in plant-animal interaction assemblages. - Oikos 113: 174-184.

MacArthur, R. H. and Wilson, E. O. 1967. The theory of island biogeography. - Princeton Univ. Press.
Menke, S. et al. 2012. Plant-frugivore networks are less specialized and more robust at forest-farmland edges than in the interior of a tropical forest. - Oikos 121: 1553-1566.

Minoarivelo, H. O. et al. 2014. Detecting phylogenetic signal in mutualistic interaction networks using a Markov process model. - Oikos 123: 1250-1260.

Minoarivelo, H. O. and Hui, C. 2016a. Invading a mutualistic network: to be or not to be similar. - Ecol. Evol. 6: 4981-4996.

Minoarivelo, H. O. and Hui, C. 2016b. Trait-mediated interaction leads to structural emergence in mutualistic networks. - Evol. Ecol. 30: 105-121.

Minoarivelo, H. O. and Hui, C. 2018. Alternative assembly processes from trait-mediated co-evolution in mutualistic communities. - J. Theor. Biol. 454: 146-153.

Montoya, D. et al. 2015. Functional group diversity increases with modularity in complex food webs. - Nat. Commun. 6: 7379.

Mougi, A. and Kondoh, M. 2016. Food-web complexity, metacommunity complexity and community stability. - Sci. Rep. 6: 24478.

Murdoch, W. W. 1969. Switching in general predators: experiments on predator specificity and stability of prey populations. - Ecol. Monogr. 39: 335-354.

Nakazawa, M. 2017. fmsb: functions for medical statistics book with some demographic data. - R package ver. 0.6.1. < https:// CRAN.R-project.org/package $=\mathrm{fmsb}>$.

Nielsen, A. and Bascompte, J. 2007. Ecological networks, nestedness and sampling effort. - J. Ecol. 95: 1134-1141.

Nuwagaba, S. et al. 2015. A hybrid behavioural rule of adaptation and drift explains the emergent architecture of antagonistic networks. - Proc. R. Soc. B 282: 20150320.

Okuyama, T. and Holland, J. N. 2008. Network structural properties mediate the stability of mutualistic communities. - Ecol. Lett. 11: 208-216.

Olesen, J. M. et al. 2007. The modularity of pollination networks. - Proc. Natl Acad. Sci. USA 104: 19891-19896.

Petanidou, T. et al. 2008. Long-term observation of a pollination network: fluctuation in species and interactions, relative invariance of network structure and implications for estimates of specialization. - Ecol. Lett. 11: 564-575.

Pilosof, S. et al. 2017. The multilayer nature of ecological networks. - Nat. Ecol. Evol. 1: 1-9.

Pinheiro, R. B. P. et al. 2016. Tradeoffs and resource breadth processes as drivers of performance and specificity in a hostparasite system: a new integrative hypothesis. - Int. J. Parasitol. 46: 115-121.

Ponisio, L. C. et al. 2017. Opportunistic attachment assembles plant-pollinator networks. - Ecol. Lett. 20: 1261-1272.

Poulakakis, N. et al. 2012. Unravelling the peculiarities of island life: vicariance, dispersal and the diversification of the extinct and extant giant Galápagos tortoises. - Mol. Ecol. 21: 160-173.

Raimundo, R. L. et al. 2018. Adaptive networks for restoration ecology. - Trends Ecol. Evol. 33: 664-675.

Ramos-Jiliberto, R. et al. 2012. Topological plasticity increases robustness of mutualistic networks. - J. Anim. Ecol. 81: 896-904.

Rezende, E. L. et al. 2007a. Effects of phenotypic complementarity and phylogeny on the nested structure of mutualistic networks. - Oikos 116: 1919-1929.

Rezende, E. L. et al. 2007b. Non-random coextinctions in phylogenetically structured mutualistic networks. - Nature 448: 925-928. 
Ricklefs, R. E. and Lovette, I. J. 1999. The roles of island area per se and habitat diversity in the species-area relationships of four Lesser Antillean faunal groups. - J. Anim. Ecol. 68: 1142-1160.

Rivera-Hutinel, A. et al. 2012. Effects of sampling completeness on the structure of plant-pollinator networks. - Ecology 93: 1593-1603.

Roura-Pascual, N. et al. 2016. The distribution and diversity of insular ants: do exotic species play by different rules? - Global Ecol. Biogeogr. 25: 642-654.

Saul, W. C. and Jeschke, J. M. 2015. Eco-evolutionary experience in novel species interactions. - Ecol. Lett. 18: 236-245.

Sebastián-González, E. et al. 2015. Macroecological trends in nestedness and modularity of seed-dispersal networks: human impact matters. - Global Ecol. Biogeogr. 24: 293-303.

Soetaert, K. et al. 2010. Solving differential equations in R: package deSolve. - J. Stat. Softw. 33: 1-25.

Stang, M. et al. 2006. Size constraints and flower abundance determine the number of interactions in a plant-flower visitor web. - Oikos 112: 111-121.

Staniczenko, P. P. A. et al. 2010. Structural dynamics and robustness of food webs. - Ecol. Lett. 13: 891-899.

Stephens, D. W. and Krebs, J. R. 1986. Foraging theory. - Princeton Univ. Press.

Suweis, S. et al. 2013. Emergence of structural and dynamical properties of ecological mutualistic networks. - Nature 500: 449-452.

Thébault, E. and Fontaine, C. 2010. Stability of ecological communities and the architecture of mutualistic and trophic networks. - Science 329: 853-856.

Timóteo, S. et al. 2018. Multilayer networks reveal the spatial structure of seed-dispersal interactions across the Great Rift landscapes. - Nat. Commun. 9: 140.

Traveset, A. et al. 2013. Invaders of pollination networks in the Galapagos Islands: emergence of novel communities. - Proc. R. Soc. B 280: 20123040.

Supplementary material (available online as Appendix oik06053 at <www.oikosjournal.org/appendix/oik-06053>). Appendix 1-5.
Traveset, A. et al. 2016. Global patterns of mainland and insular pollination networks. - Global Ecol. Biogeogr. 25: 880-890.

Trøjelsgaard, K. and Olesen, J. M. 2013. Macroecology of pollination networks. - Global Ecol. Biogeogr. 22: 149-162.

Tylianakis, J. M. and Morris, R. J. 2017. Ecological networks across environmental gradients. - Annu. Rev. Ecol. Evol. Syst. 48: 25-48.

Valdovinos, F. S. et al. 2010. Consequences of adaptive behaviour for the structure and dynamics of food webs. - Ecol. Lett. 13: 1546-1559.

van Baalen, M. et al. 2001. Alternative food, switching predators and the persistence of predator-prey systems. - Am. Nat. 157: $512-524$.

Vázquez, D. P. et al. 2007. Species abundance and asymmetric interaction strength in ecological networks. - Oikos 116: 1120-1127.

Vizentin-Bugoni, J. et al. 2015. Influences of sampling effort on detected patterns and structuring processes of a Neotropical plant-hummingbird network. - J. Anim. Ecol. 85: 262-272.

Wallace, A. R. 1864. The origin of human races and the antiquity of man deduced from the theory of 'natural selection'. - J. Anthropol. Soc. Lond. 2: clviii-clxxxvii.

Walsh, C. and Mac Nally, R. 2013. hier.part: hierarchical partitioning. - $\mathrm{R}$ package ver. 1.0-4. <https:/CRAN.R-project.org/ package $=$ hier. part $>$.

Whittall, J. B. and Hodges, S. A. 2007. Pollinator shifts drive increasingly long nectar spurs in columbine flowers. - Nature 447: 706-709.

Zhang, F. and Hui, C. 2014. Recent experience-driven behaviour optimizes foraging. - Anim. Behav. 88: 13-19.

Zhang, F. et al. 2011. An interaction switch predicts the nested architecture of mutualistic networks. - Ecol. Lett. 14: 797-803. 